\title{
Contributions to the thermophilous fringe communities (Trifolio-Geranietea sanguinei) in Belarus
}

\author{
Dmytro Iakushenko
}

Faculty of Biological Sciences, University of Zielona Góra, Z. Szafrana 1, 65-516 Zielona Góra, Poland

e-mail: d.iakushenko@wnb.uz.zgora.pl

\begin{abstract}
The paper presents the results of phytosociological studies on thermophilous forb fringes conducted in August, 2012, in Belarus. A dataset of 31 relevés is analyzed, 3 associations and 1 community of 3 alliances and 3 orders of the class Trifolio-Geranietea sanguinei T. Müller 1962 are distinguished. For the first time for this area, the association Galio borealisGeranietum sanguinei Tüxen 1967 is reported and characterized. The results of syntaxonomical interpretation are discussed based on chorological data.
\end{abstract}

Key words: forest fringes; edges, Trifolio-Geranietea sanguinei; syntaxonomy; vegetation; Belarus

\section{Introduction}

Thermophilous fringe communities on forest edges and margins formed by meso-xerothermic forbs are widespread in forest zone of Europe. These marginal plant communities are considered within distinct Trifolio-Geranietea sanguinei T. Müller 1962 class (Müller 1962; Dierschke 1974a; Mucina 1997). The communities of this class have been studied since 1960s in different parts of the continent, also in Central-Eastern Europe (Dierschke 1974b; Passarge 1979; Mucina \& Kolbek 1993; Brzeg 2005; Čarni 2005; Chytrý 2007; Brzeg \& Wika 2014; Valachovič \& Hegedüšová Vantarová 2014).

However, for the territory of Belarus, communities of the Trifolio-Geranietea sanguinei class were mentioned only scarcely. Mesophillous fringes dominated by Trifolium medium were characterized based on 10 phytosociological relevés from north-western part of the country (Scepanovich \& Scepanovich 1991). In the last vegetation survey, 3 associations of this class were listed: Stachyo-Melampyretum nemorosi Passarge 1967 (within the Melampyrion pratensis Passarge 1967 alliance), Trifolietum medii Müller $1961 \mathrm{em}$. Stepanovič (1987) 1991 and Equisetetum hyemali ass. nova (in the Trifolion medii Müller 1961 alliance) (Scepanovich 2006). Xero-thermophilous edges were neglected or omitted, only for south-eastern part of the country, one relevé classified as Geranio-Trifolietum alpestris T. Müller 1962 galietosum aparines Korneck 1974 was published (Lukash \& Iakushenko 2008).

So, the thermophilous edge vegetation in Belarus is poorly documented and still studied fragmentarily. The aim of this paper was to present new phytosociological materials and syntaxonomical considerations on some forest fringe communities from Belarus.

\section{Material and methods}

\subsection{Study area}

The natural forest vegetation of the country is mainly represented by hemiboreal spruce forests with broadleaved trees and by hemiboreal Scots-pine forests (Bohn et al. 2000). Belarus is divided into three vegetation subzones: the oak-spruce forests sub-zone in the northern part, the hornbeam-oak-spruce forests sub-zone in the central part, and the broadleaved-pine forests sub-zone in the southern part of the country (Yurkevich et al. 1979). The first sub-zone mainly overlaps with the Boreal biogeographical region and two others - with the Continental region (Evans 2005).

The data were sampled in the geobotanical region Ashmyanska-Minskaya akruga of the oak-spruce forests sub-zone, on three geomorphological units: (1) Narachanskaya plain, (2) Svyantsyanskiya ridges (two units - within the frames of the Narachanski National 


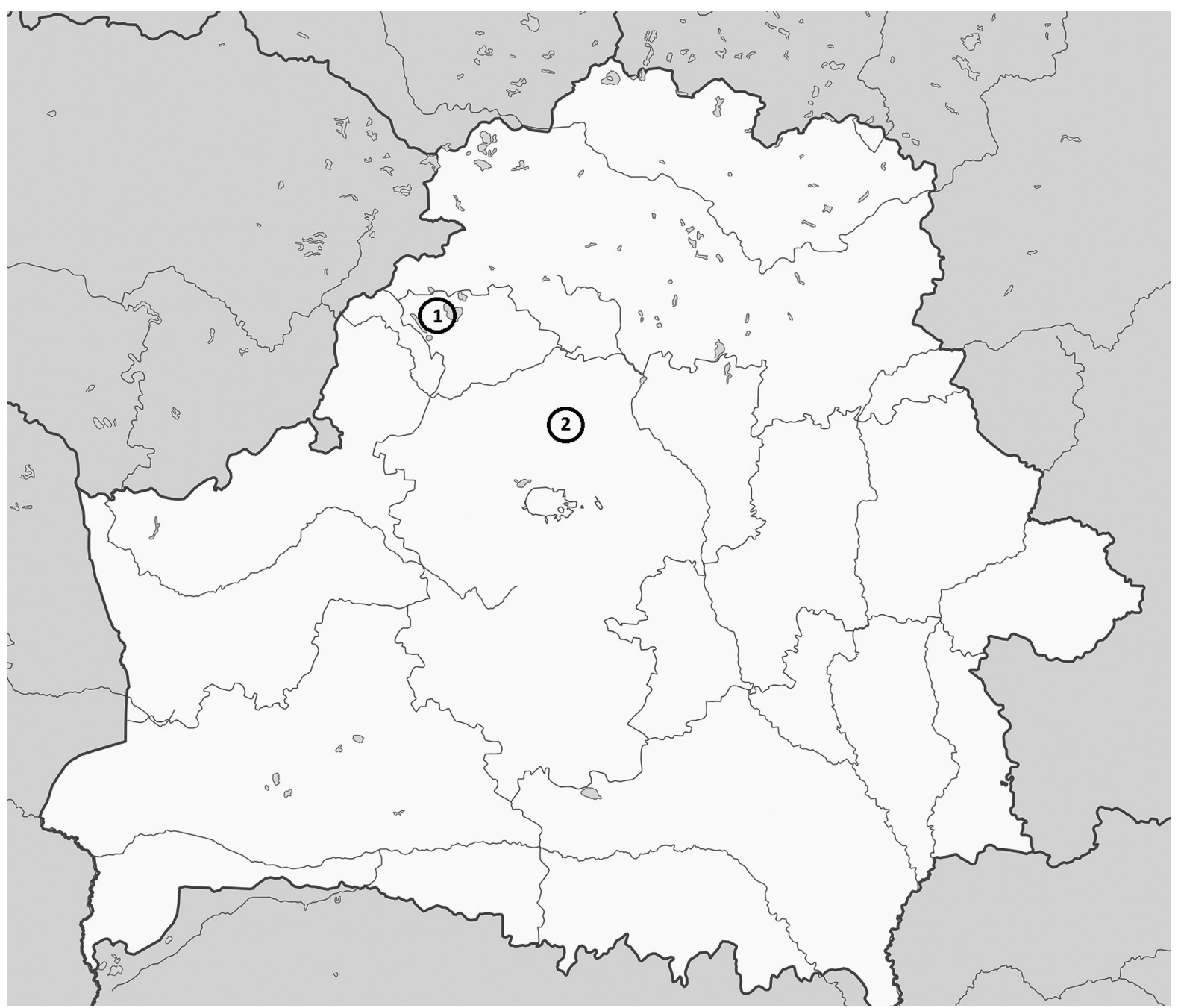

Fig. 1. Map of Belarus with studied localities (https://commons.wikimedia.org/wiki/File:Belarus_location_map.svg)

Explanations: 1 - Narachanski National Park, Miadziel district, Minsk region, 2 - vicinities of Kozyry village, Lahoysk district, Minsk region

Park), and (3) Minskaya highland (Fig. 1) (Myasnikowich 2002).

\subsection{Data collection and phytosociological analysis}

The research was conducted in the middle of August, 2012. The phytosociological relevé sampling followed the Braun-Blanquet approach (Westhoff \& van der Maarel 1973). The relevés were stored in the TurboVeg database (Hennekens \& Schaminée 2001). A small dataset of 31 relevés was used for the analysis using Juice 7.0 software package (Tichý 2002). Numerical classification of the dataset was performed by the PC-ORD 5 program (McCune \& Mefford 1999) with relative Euclidean distance as a measure of dissimilarity and Ward's linkage method, square-root transformed cover values were used.

Diagnostic species were chosen on the basis of fidelity measure (Chytrý et al. 2002). The threshold value for a species considered as diagnostic was set at a Phi-coefficient (multiplied by 100) more than
30. Species that were recorded in at least $61 \%$ of the relevés of a community were considered constant for a syntaxon. The classification followed the results of syntaxonomical revisions of the Trifolio-Geranietea sanguinei class for adjacent European countries: Latvia (Rūsiņa 2007), Lithuania (Balevičiene et al. 1998), Poland (Brzeg 2005), and Ukraine (Solomakha 2008). The names of syntaxa and lists of diagnostic species were based on the current European synthesis (Mucina et al. 2016). The nomenclature of vascular plants followed the Euro+Med PlantBase (Euro+Med 2006-), and for mosses - a checklist by Hill et al. (2006).

\section{Results}

\subsection{Classification of plant communities}

The results of cluster analysis are presented in the dendrogram (Fig. 2). As a result of PC-ORD analysis, six distinct groups of relevés were obtained. In two main clusters, the first one consisted of the communities asso- 


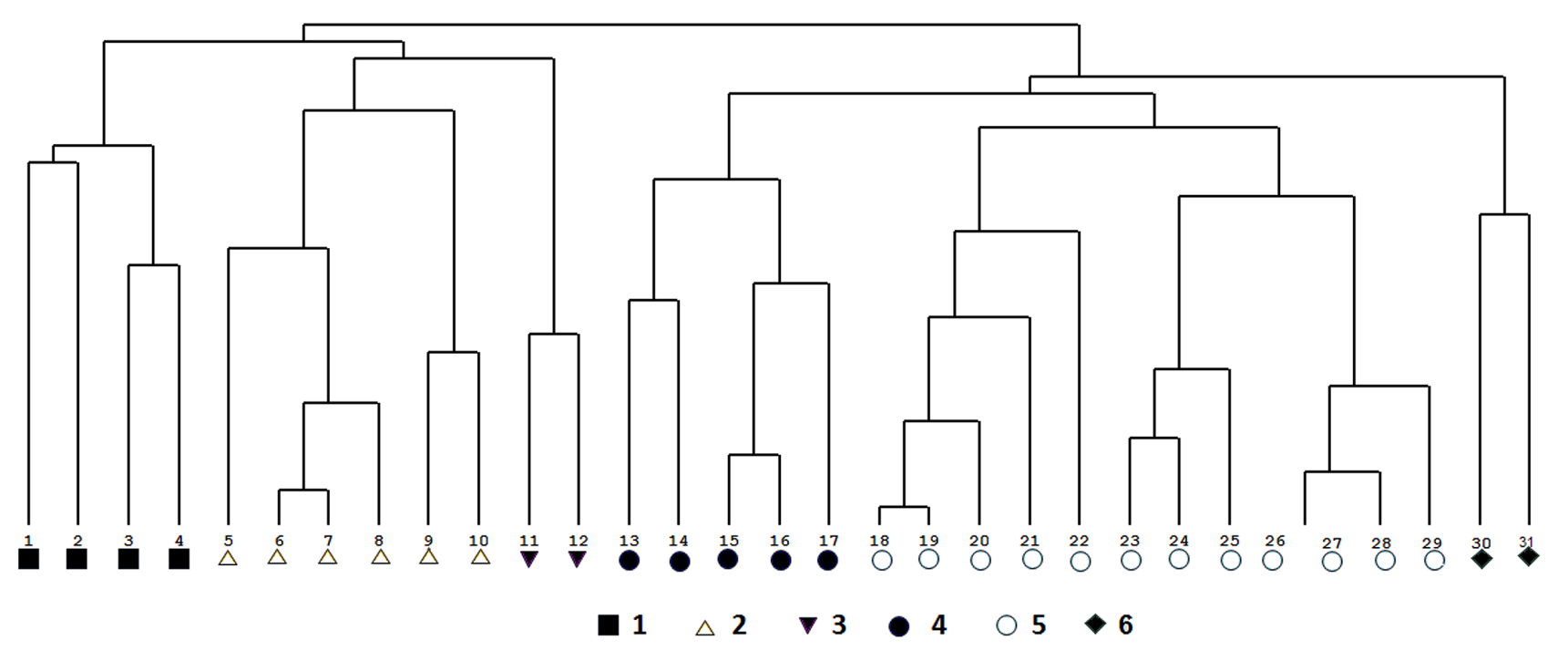

Fig. 2. Dendrogram of numerical classification of the studied relevés

Explanations: 1 - Galio borealis-Geranietum sanguinei, 2 - community with Melampyrum pratense - typical plots, 3 - community with $M$. pratense - plots dominated by Vicia cassubica, 4 - Trifolio-Melampyretum nemorosi-thermophilous plots, 5 -Trifolio-Melampyretum nemorosi, 6 -Trifolio medii-Agrimonietum

ciated with the edges of coniferous (pine or spruce-pine) forests. The further division within this cluster reflected the differentiation into xerothermophilous communities (group 1) and acidophilous communities on sandy soils (groups 2-3). The second main cluster consisted of mesophilous fringe communities (groups 4-6).

For these three clusters, the constancy and threshold value for a diagnostic species are presented in Table 1. The most frequent herbal species with high fidelity for the first group were Calamagrostis arundinacea, Festuca ovina, Fragaria vesca, Geranium sanguineum, Hieracium umbellatum, Peucedanum oreoselinum, Polygonatum odoratum, Pulmonaria angustifolia, Rubus saxatilis, and Veronica spicata. This group corresponds to the Geranion sanguinei alliance. For the second group, which refers to the Melampyrion pratensis alliance, such species as Achillea millefolium, Knautia arvensis, Melampyrum pratense, and Solidago virgaurea, were the most frequent with high fidelity index. Dactylis glomerata and Trifolium medium were the most frequent species with high fidelity in the third cluster, corresponding to the Trifolion medii alliance.

As a result of syntaxonomical interpretation of phytosociological materials, the following scheme was established:

Trifolio-Geranietea sanguinei T. Müller 1962

Antherico ramosi-Geranietalia sanguinei Julve ex

Dengler in Dengler et al. 2003

Geranion sanguinei Tx. in T. Müller 1962

Galio borealis-Geranietum sanguinei Tüxen 1967

Melampyro-Holcetalia mollis Passarge in Theurillat et al. 1995
Melampyrion pratensis Passarge 1979

Community with Melampyrum pratense

Origanetalia vulgaris T. Müller 1962

Trifolion medii T. Müller 1962

Trifolio-Melampyretum nemorosi (Passarge 1967) Dierschke 1973

Trifolio medii-Agrimonietum T. Müller 1962

\subsection{Characteristics of the associations}

Galio borealis-Geranietum sanguinei Tüxen 1967 association (Table 2): Species-rich (average number of species per relevé was 45.5), dense (herb layer cover consisted of $80-95 \%$ ), picturesque communities dominated by forbs: Laserpitium latifolium (20-25\%), Geranium sanguineum (10-30\%), Vincetoxicum hirundinaria (5-15\%), Clinopodium vulgare (10-15\%), Dracocephalum ruyschiana (up to 10\%), Convallaria majalis, Rubus saxatilis, Galium boreale, Polygonatum odoratum, Lathyrus niger, etc. and broad-leaved graminoids: Calamagrostis arundinacea (5-15\%), Brachypodium pinnatum (up to 30\%), Molinia caerulea $(15-17 \%)$, Carex montana (up to $20 \%$ ). The species of the Trifolio-Geranietea class and the Geranion sanguinei alliance were well represented. A significant role of the species from the Molinion caerulei alliance (Molinia caerulea, Laserpitium prutenicum, Rhinanthus serotinus, and Succisa pratensis) should be admitted. Also, the described plots were rich in species protected on national level (Kachanovskiy et al. 2015): Dracocephalum ruyschiana, Laserpitium latifolium, Lathyrus pisiformis, Pulsatilla patens, and Thesium ebracteatum. In the shrub layer (cover 5\%), 
Table 1. Shortened synoptic table with frequency (\%) and modified fidelity index for the distinguished alliances*

\begin{tabular}{|c|c|c|c|}
\hline Successive No. & 1 & 2 & 3 \\
\hline Number of relevés & 4 & 8 & 19 \\
\hline Total number of species & 84 & 87 & 155 \\
\hline \multicolumn{4}{|l|}{ D.s. Al. Geranion sanguinei } \\
\hline Geranium sanguineum & $100^{100.0}$ &.-- &.-- \\
\hline Polygonatum odoratum & $100^{100.0}$ & --- &.-- \\
\hline Pulmonaria angustifolia & $100^{100.0}$ & -- & -- \\
\hline Veronica spicata** & $100^{100.0}$ & -- & -- \\
\hline Rubus saxatilis** & $100^{100.0}$ & --- & $\cdot-$ \\
\hline Calamagrostis arundinacea** & $100^{92.6}$ & -- & $\dot{11}--$ \\
\hline Vincetoxicum hirundinaria & $75^{81.6}$ & --- &.-- \\
\hline Dracocephalum ruyschiana & $75^{81.6}$ & -- & -- \\
\hline Lathyrus niger & $75^{81.6}$ & --- & -- \\
\hline Galium boreale & $75^{81.6}$ & -- & -- \\
\hline Carex montana & $75^{81.6}$ & --- & -- \\
\hline Convallaria majalis** & $75^{81.6}$ & -- & -- \\
\hline Viola rupestris & $75^{81.6}$ & --- & --- \\
\hline Laserpitium prutenicum $* *$ & $75^{81.6}$ & -- & 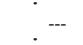 \\
\hline Rhinanthus serotinus $* *$ & $75^{81.6}$ & --- & --- \\
\hline \multicolumn{4}{|l|}{ D.s. Al. Melampyrion pratensis } \\
\hline Melampyrum pratense & $25--$ & $88^{73.0}$ &.-- \\
\hline Luzula pilosa $* *$ &.-- & $62^{51.0}$ & $26^{---}$ \\
\hline \multicolumn{4}{|l|}{ D.s. Al. Trifolion medii } \\
\hline Dactylis glomerata** &.-- & $88^{38.7}$ & $95^{49.2}$ \\
\hline Trifolium medium &.-- & $25^{--}$ & $89^{74.7}$ \\
\hline Galium mollugo** & $25--$ & $62^{12.4}$ & $74^{28.3}$ \\
\hline \multicolumn{4}{|l|}{ D.s. CI. Trifolio-Geranietea } \\
\hline Peucedanum oreoselinum & $100^{80.7}$ & $25^{--}$ & $5^{--}$ \\
\hline Hieracium umbellatum & $100^{63.1}$ & $25^{--}$ & $42-$ \\
\hline Fragaria vesca & $100^{40.6}$ & $62-$ & $63^{---}$ \\
\hline Campanula persicifolia & $75^{77.1}$ &.-- & $5-$ \\
\hline Trifolium alpestre & $75^{72.8}$ & 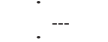 & 11 \\
\hline Clinopodium vulgare & $75^{63.8}$ & $12^{---}$ & 11 \\
\hline Solidago virgaurea & $75^{---}$ & $100^{38.0}$ & 58 \\
\hline Knautia arvensis & $75-$ & $100^{28.0}$ & 84 \\
\hline Veronica chamaedrys & 75 & $88^{9.7}$ & $84^{3.7}$ \\
\hline Melampyrum nemorosum & $75^{22.1}$ & $25--$ & $79^{27.8}$ \\
\hline Hypericum perforatum & $50^{10.3}$ & $62^{28.2}$ & $16^{--}$ \\
\hline Poa angustifolia & $25--$ & $62^{38.7}$ & $21-$ \\
\hline \multicolumn{4}{|l|}{ Other species } \\
\hline Festuca ovina & $100^{74.2}$ & $38^{--}$ & $5--$ \\
\hline Ajuga reptans & $75^{61.6}$ &.-- & $26-$ \\
\hline Vaccinium vitis-idaea & $75^{55.9}$ & $25--$ & 11 \\
\hline Agrostis capillaris & $75^{12.2}$ & $62-$ & $63^{---}$ \\
\hline Achillea millefolium & $50^{---}$ & $100^{47.1}$ & $58--$ \\
\hline Lupinus polyphyllus &.- & $62^{62.9}$ & 11 \\
\hline Artemisia vulgaris &.- & $62^{35.1}$ & $53^{20.7}$ \\
\hline Pimpinella saxifraga & 25 & $50^{3.1}$ & $68^{29.2}$ \\
\hline Phleum pratense &.-- & $38^{3.2}$ & $68^{49.0}$ \\
\hline Vicia cracca & -- & $25^{--}$ & $74^{61.4}$ \\
\hline
\end{tabular}

Explanations: 1 - Geranion sanguinei (Table 2), 2-Melampyrion pratensis (Table 3), 3 - Trifolion medii (Table 4); * - Only species with constancy of more than $61 \%$ at least in one column, are shown. Species from the layers $b$ and $d$ are excluded; ** - Species which are diagnostic to other syntaxa, but obtained a high frequency and fidelity in this dataset

Picea abies, Juniperus communis, Pinus sylvestris, Sorbus aucuparia were common. Moss layer (cover up to $20 \%$ ) was formed by Pleurozium schreberi, Hylocomium splendens, Dicranum polysetum, Polytrichum juniperinum. The communities of the association were formed on forest glades within species-rich pine forests on gentle slopes $\left(10-20^{\circ}\right)$ of moraine hills with sandy-clay soils. Diagnostic species: Geranium sanguineum (opt.), Galium boreale, Dracocephalum ruyschiana.

Community with Melampyrum pratense (Table 3): These communities were rather sparse (cover from 30 to $60 \%$, up to $85 \%$ ) and well recognized physiognomically by the aspect of Melampyrum pratense (cover 10-40\%). In relatively poor species composition (average number of species per relevé was 28.5), grasses obtained a sig- 
Table 2. Floristic composition of communities from the alliance Geranion sanguinei

\begin{tabular}{lcccc}
\hline Successive No. of relevé & 1 & 2 & 3 & 4 \\
No. of relevé in the field & 72 & 73 & 74 & 35 \\
Shrub layer cover [\%] & 5 & 5 & 5 & 5 \\
Herb layer cover [\%] & 80 & 95 & 85 & 80 \\
Moss layer cover [\%] & 20 & 5 & 20 & 3 \\
Altitude a.s.1. [m] & 256 & 260 & 258 & 197 \\
Slope exposure & $\mathrm{NW}$ &. & $\mathrm{S}$ & $\mathrm{S}$ \\
Inclination [ [ ] & 20 &. & 10 & 10 \\
Area of relevé [m2] & 100 & 100 & 100 & 30 \\
Number of species & 48 & 50 & 43 & 41 \\
\hline
\end{tabular}

D.s. Ass. Galio borealis-Geranietum sanguinei

Geranium sanguineum

Galium boreale

Dracocephalum ruyschiana

D.s. Al. Geranion sanguinei

Polygonatum odoratum

Veronica spicata

Calamagrostis arundinacea

Pulmonaria angustifolia

Rubus saxatilis

Vincetoxicum hirundinaria

Carex montana

Lathyrus niger

Viola rupestris

Laserpitium latifolium

Brachypodium pinnatum

D.s. Cl. Trifolio-Geranietea

Hieracium umbellatum

Peucedanum oreoselinum

Fragaria vesca

Veronica chamaedrys

Trifolium alpestre

Campanula persicifolia

Solidago virgaurea

Knautia arvensis

Melampyrum nemorosum

Clinopodium vulgare

Ranunculus polyanthemos

Lathyrus pisiformis

Hypericum perforatum

Silene nutans

D.s. Cl. Vaccinio-Piceetea

Pleurozium schreberi d

Vaccinium vitis-idaea

Dicranum polysetum d

Picea abies b

Juniperus communis b

Vaccinium myrtillus

D.s. Cl. Nardetea strictae

Potentilla erecta

Veronica officinalis

Calluna vulgaris

D.s. Cl. Koelerio-Corynephoretea

Thymus serpyllum

Pilosella officinarum

D.s. Al. Molinion caeruleae

Laserpitium prutenicum

Rhinanthus serotinus

Molinia caerulea

D.s. Cl. Carpino-Fagetea

Melica nutans

Carex digitata

Convallaria majalis

Other species

Festuca ovina

Pinus sylvestris b

Agrostis capillaris

Sorbus aucuparia b

Ajuga reptans

Pteridium aquilinum

Populus tremula b

Achillea millefolium nificant role (Agrostis capillaris, Festuca ovina, Holcus mollis, Anthoxanthum odoratum, Dactylis glomerata, Poa angustifolia, etc.). Among dicots, mesophilous and slightly psammophytic species prevailed (Veronica chamaedrys, Knautia arvensis, Pilosella officinarum, Solidago virgaurea, Achillea millefolium, Fragaria vesca, etc.). The advanced invasion of Lupinus polyphyllus was observed. Two stands (Table 3, rel. 7-8) were strongly dominated by Vicia cassubica (cover of this species - up to $50 \%$, total herb cover varied in 2 relevés from 50 to $70 \%$ ). Moss layer normally was developed (cover up to $70-80 \%$ ), formed by typical forest mosses: Hylocomium splendens and Pleurozium schreberi. Communities with Melampyrum pratense formed on narrow strip linear margins of pine forests on sandy soils (Dicrano-Pinion sylvestris (Libbert 1933) W. Matuszkiewicz 1962). Diagnostic species: Melampyrum pratense (opt.), Agrostis capillaris, Holcus mollis, Festuca ovina.

Trifolio-Melampyretum nemorosi (Passarge 1967) Dierschke 1973 association (Table 4, rel. 1-17): These dense (total cover varied from 70 to $100 \%$ ) communities commonly distinguished physiognomically by the co-domination in different proportions of two mesophilous species: Melampyrum nemorosum and Trifolium medium. Other species characteristic for the Trifolion medii alliance (Galium mollugo, Dactylis glomerata, Vicia sepium, etc.) and for the Trifolio-Geranietea class (Solidago virgaurea, Veronica chamaedrys, Knautia arvensis, Hieracium umbellatum) were frequent. In species composition, various species of mesophytic meadows (Phleum pratense, Vicia cracca, Centaurea jacea, etc.) and ruderal habitats (Anthriscus sylvestris, Artemisia vulgaris, Geum urbanum, Cirsium arvense, etc.) were common. Some sites were enriched in xeromesophilous species from Festuco-Brometea Br.-B1. et Tx. ex Soó 1947 class: Centaurea scabiosa, Medicago
Sporadic taxa: rel. 1: Hylocomium splendens d (2), Lathyrus vernus (r), Orthilia secunda (1), Pimpinella saxifraga (r), Polytrichum juniperinum d (1); rel. 2: Astragalus glycyphyllos (1), Cirsium arvense (1), Galium mollugo (r), Succisa pratensis (r), Urtica dioica (r); rel. 3: Betula pendula b (1), Gnaphalium sylvaticum (1), Koeleria grandis (1), Rosa sp. b (1), Sieglingia decumbens (r), Thesium ebracteatum (1), Vicia cassubica (1); rel. 4: Anthyllis vulneraria (1), Astragalus arenarius (1), Carex ericetorum (1), Erigeron acris (r), Hylotelephium maximum (1), Hypochaeris maculata (1), Hypochaeris radicata (1), Melampytum pratense (1), Poa angustifolia (1), Pulsatilla patens (1), Rubus idaeus (r), Scorzonera humilis (1), Verbascum thapsus (r), Viola canina (r)

\section{List of localities:}

1 - 54 $19.362^{\prime}$ N 2755.639' E; Minsk Region, Lahoysk district, $4.5 \mathrm{~km}$ E of Kazyry village; 19.08.2012; 2 - 54¹9.299’ N 2755.049’ E; Minsk Region, Lahoysk district, $4.5 \mathrm{~km}$ E of Kazyry village; 19.08.2012; 3 - 54²19.274' N $27^{\circ} 55.037^{\prime}$ E; Minsk Region, Lahoysk district, 4.5 km E of Kazyry village; 19.08.2012; 4 - 54 $4^{\circ} 57.932^{\prime}$ N 26²3.080’ E; Minsk Region, Miadziel district, vicinities of Glublia lake; 15.08.2012 
Table 3. Floristic composition of communities from the alliance Melampyrion pratensis

\begin{tabular}{|c|c|c|c|c|c|c|c|c|c|}
\hline Successive No. of relevé & 1 & 2 & 3 & 4 & 5 & 6 & 7 & 8 & \\
\hline No. of relevé in the field & 30 & 33 & 31 & 15 & 62 & 61 & 32 & 68 & \\
\hline Shrub layer cover $[\%]$ & 3 & 5 & 1 & 1 & 5 & 5 & 10 & 1 & \\
\hline Herb layer cover [\%] & 30 & 60 & 30 & 85 & 60 & 60 & 70 & 50 & छ్ \\
\hline Moss layer cover [\%] & 50 & 10 & 5 & . & 70 & 80 & 5 & . & $\tilde{0}$ \\
\hline Altitude a.s.l. [m] & 206 & 206 & 206 & 173 & 172 & 171 & 206 & 144 & \\
\hline Area of relevé [m2] & 20 & 50 & 50 & 20 & 100 & 40 & 25 & 50 & \\
\hline Number of species & 33 & 33 & 25 & 15 & 35 & 33 & 29 & 25 & \\
\hline
\end{tabular}

D.s. Al. Melampyrion pratense Melampyrum pratense Holcus mollis Anthoxantum odoratum

\begin{tabular}{|llllllll|l|}
\hline 2 & 3 & 2 & 5 & 4 & 3 &. & 1 & V \\
1 & 1 & 3 &. &. &. & 1 &. & III \\
1 & 1 & 1 &. &. &. & 1 &. & III
\end{tabular}

D.s. Al. Trifolion medii

Dactylis glomerata

Galium mollugo

Trifolium medium

D.s. Cl. Trifolio-Geranietea

Solidago virgaurea

Veronica chamaedrys

Knautia arvensis

Fragaria vesca

Hypericum perforatum

Poa angustifolia

Peucedanum oreoselinum

Melampyrum nemorosum

Ranunculus polyanthemos

Silene nutans

Hieracium umbellatum

Vicia cassubica

$\begin{array}{ccccccccc}. & 1 & 2 & 1 & 1 & 2 & 1 & 1 & \mathrm{~V} \\ . & 1 & . & 1 & \mathrm{r} & 1 & . & 1 & \mathrm{IV} \\ \mathrm{r} & 1 & . & . & . & . & . & . & \mathrm{II}\end{array}$

D.s. Cl. Molinio-Arrhenateretea

Phleum pratense

Poa pratensis

Equisetum pratense

Taraxacum officinale

Rumex acetosa

Vicia cracca

D.s. Cl. Vaccinio-Piceetea

Pleurozium schreberi d

Luzula pilosa

Hylocomium splendens d

Vaccinium vitis-idaea

Vaccinium myrtillus

Trientalis europaea

Polytrichum juniperinum $\mathrm{d}$

\begin{tabular}{|cccccccc|c}
\hline 1 & 1 & 1 & $\mathrm{r}$ & 1 & $\mathrm{r}$ & 2 & 1 & $\mathrm{~V}$ \\
1 & 1 & 1 & 2 & 1 & 2 &. & 1 & $\mathrm{~V}$ \\
2 & 1 & 1 & 1 & 1 & 1 & 1 & 2 & $\mathrm{~V}$ \\
1 & 1 &. &. & 1 & 3 & 3 &. & $\mathrm{IV}$ \\
$\mathrm{r}$ & 1 & 1 &. & $\mathrm{r}$ &. & $\mathrm{r}$ &. & $\mathrm{IV}$ \\
. & 1 &. & 1 & 1 & 1 &. & 1 & $\mathrm{IV}$ \\
. &. &. &. & $\mathrm{r}$ & 2 &. &. & $\mathrm{II}$ \\
. &. &. &. & 1 & 1 &. &. & $\mathrm{II}$ \\
. &. &. &. & $\mathrm{r}$ & $\mathrm{r}$ &. & $\mathrm{r}$ & $\mathrm{II}$ \\
. &. &. &. & 1 & 1 &. &. & $\mathrm{II}$ \\
. & $\mathrm{r}$ &. &. &. &. & $\mathrm{r}$ &. & $\mathrm{II}$ \\
. &. &. &. &. &. & 5 & 4 & $\mathrm{II}$
\end{tabular}

D.s. Cl. Nardetea strictae

Veronica officinalis

Calluna vulgaris

D.s. Cl. Koelerio-Corynephoretea

Pilosella officinarum

Jasione montana

Rumex acetosella

D.s. Cl. Artemisietea vulgaris

Artemisia vulgaris

Elytrigia repens

Tanacetum vulgare

Silene latifolia

D.s. Cl. Carpino-Fagetea

Acer platanoides $\mathrm{b}$

\begin{tabular}{|c|c|c|c|c|c|c|}
\hline $\mathrm{r}$ & . & . & . & . & 1 & $\mathrm{r}$ \\
\hline 1 & . & . & . & . & . & 1 \\
\hline - & 1 & . & . & . & $\mathrm{r}$ & $\mathrm{r}$ \\
\hline . & $\mathrm{r}$ & . & . & $\mathrm{r}$ & - & $\mathrm{r}$ \\
\hline . & . & 1 & . & 1 & . & $\mathrm{r}$ \\
\hline & . & . & $\mathrm{r}$ & $\mathrm{r}$ & . & . \\
\hline
\end{tabular}




\begin{tabular}{|c|c|c|c|c|c|c|c|c|c|}
\hline Other species & & & & & & & & & \\
\hline Achillea millefolium & 1 & 2 & 2 & $\mathrm{r}$ & 1 & $\mathrm{r}$ & 1 & $\mathrm{r}$ & $\mathrm{V}$ \\
\hline Agrostis capillaris & 1 & 2 & . & 2 & 3 & 3 & . & . & IV \\
\hline Lupinus polyphyllus & 1 & 2 & 1 & 1 & . & . & . & 1 & IV \\
\hline Pimpinella saxifraga & . & $\mathrm{r}$ & . & $\mathrm{r}$ & . & 1 & . & $\mathrm{r}$ & III \\
\hline Rubus idaeus & . & 1 & 1 & . & 1 & 1 & . & . & III \\
\hline Salix caprea b & 1 & 1 & . & . & . & . & . & . & II \\
\hline Festuca ovina & 2 & 1 & . & . & 3 & . & . & . & II \\
\hline Populus tremula b & $\mathrm{r}$ & 1 & . & . & 1 & . & . & . & II \\
\hline Pinus sylvestris b & 1 & 1 & . & . & . & . & 2 & . & II \\
\hline Pteridium aquilinum & . & 2 & $\mathrm{r}$ & . & . & . & . & . & II \\
\hline Erigeron acris & . & . & 1 & . & . & . & 1 & . & II \\
\hline Betula pendula $\mathrm{b}$ & . & . & . & . & 1 & . & 1 & - & II \\
\hline Sorbus aucuparia b & . & . & . & . & 1 & 1 & . & . & II \\
\hline Quercus robur b & . & . & . & . & 1 & 1 & . & . & II \\
\hline Quercus robur c & $\mathrm{r}$ & . & . & . & . & . & . & . & I \\
\hline Malus domestica b & . & . & . & . & . & 1 & 1 & . & II \\
\hline Medicago sativa & . & . & . & . & . & 1 & . & 1 & II \\
\hline
\end{tabular}

Sporadic taxa: rel. 1: Galeopsis bifida (r), Luzula campestris (r), Trifolium repens (1); rel. 2: Artemisia campestris (r), Equisetum pratense (1), Orthilia secunda (1); rel. 3: Convolvulus arvensis (1), Frangula alnus b (r), Hypochaeris radicata (1); rel. 4: Amelanchier spicata b (1); rel. 5: Chamaenerion angustifolium (r), Dicranum polysetum d (2), Picea abies b (r), Sieglingia decumbens (1); rel. 6: Agrimonia eupatoria (r), Carex hirta (1), Centaurea scabiosa (r), Euphorbia cyparissias (r), Melica nutans (r), Potentilla argentea (r); rel. 7: Carlina sp. (r), Clinopodium vulgare (1), Epilobium sp. (1), Gnaphalium sylvaticum (r), Potentilla erecta (1); rel. 8: Schoenodorus pratensis (r), Linaria vulgaris (1), Oenothera rubricaulis (r), Verbascum nigrum (r), Viburnum opulus b (1)

\section{List of localities:}

1 - 545ㄱ.188’ N 2648.682’ E; Minsk Region, Miadziel district, vicinities of Grumbinenty village; $15.08 .2012 ; 2$ - 54 $56.540^{\prime}$ N 26 24.550' E; Minsk Region, Miadziel district, vicinities of Grumbinenty village; 15.08.2012; 3 - 54 $56.538^{\prime}$ N $26^{\circ} 24.539^{\prime}$ E; Minsk Region, Miadziel district, vicinities of Grumbinenty village; $15.08 .2012 ; 4$ - 54 ${ }^{\circ} 51.892^{\prime} \mathrm{N} 26^{\circ} 43.199^{\prime} \mathrm{E}$; Minsk Region, Miadziel district, vicinities of Nanosy village; 13.08.2012;
5 - 54 $54.471^{\prime}$ N 26 $6^{\circ} 42.874$ ' E; Minsk Region, Miadziel district, vicinities of Narach town; 17.08.2012; 6 - 54 $53.561^{\prime}$ N 26²41.367' E; Minsk Region, Miadziel district, vicinities of Narach town; $17.08 .2012 ; 7-54^{\circ} 56.547^{\prime} \mathrm{N}$ $26^{\circ} 24.561^{\prime} \mathrm{E}$; Minsk Region, Miadziel district, vicinities of Grumbinenty

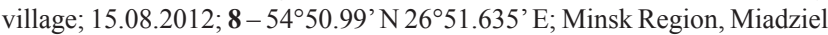
district, S of Gatavichy village; 18.08.2012 falcata, Trifolium montanum, Plantago media (Table 4, rel. 1-4). Other communities, developed in shady and moist conditions, contained nemoral forest species: Aegopodium podagraria, Stellaria holostea, Asarum europaeum. Shrubs cover up to $10 \%$. Moss layer was rare and sparse. The communities of the association were common in mesic conditions on margins and forest glades of broad-leaved and spruce-broad-leaved

Table 4. Floristic composition of communities from the alliance Trifolion medii

\begin{tabular}{|c|c|c|c|c|c|c|c|c|c|c|c|c|c|c|c|c|c|c|c|c|}
\hline Successive No. of relevé & 1 & 2 & 3 & 4 & 5 & 6 & 7 & 8 & 9 & 10 & 11 & 12 & 13 & 14 & 15 & 16 & 17 & & 18 & 19 \\
\hline No. of relevé in the field & 29 & 17 & 18 & 12 & 37 & 34 & 21 & 20 & 19 & 27 & 23 & 28 & 38 & 11 & 26 & 25 & 24 & & 64 & 36 \\
\hline Shrub layer cover $[\%]$ & 7 & & 10 & & 5 & 1 & 5 & & 10 & 4 & & 5 & 10 & 5 & 10 & 5 & 10 & 단 & 15 & 2 \\
\hline Herb layer cover [\%] & 90 & 95 & 95 & 80 & 70 & 95 & 85 & 100 & 100 & 95 & 95 & 85 & 70 & 65 & 85 & 85 & 95 & & 80 & 85 \\
\hline Moss layer cover[\%] & $\cdot$ & . & . & 5 & . & . & . & $\cdot$ & $\cdot$ & $\cdot$ & & . & 15 & . & . & . & 3 & & 10 & 10 \\
\hline Altitude a.s.1. [m] & 178 & 157 & 161 & 170 & 224 & 220 & 200 & 193 & 194 & 184 & 187 & . & 228 & 158 & 186 & 185 & 180 & 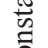 & 163 & 172 \\
\hline Inclination $\left[{ }^{\circ}\right]$ & . & $\cdot$ & $\cdot$ & $\cdot$ & $\cdot$ & $\cdot$ & $\cdot$ & $\cdot$ & $\cdot$ & 5 & $\cdot$ & . & . & . & $\cdot$ & $\cdot$ & $\cdot$ & ن & . & . \\
\hline Area of relevé [m2] & 100 & 100 & 50 & 50 & 50 & 20 & 40 & 10 & 60 & 35 & 20 & 25 & 30 & 50 & 25 & 50 & 100 & & 50 & 20 \\
\hline Number of species & 45 & 25 & 30 & 31 & 34 & 32 & 25 & 21 & 29 & 29 & 28 & 33 & 29 & 28 & 30 & 38 & 41 & & 30 & 31 \\
\hline
\end{tabular}

D.s. Ass. Trifolio medii-Agrimonietum

Agrimonia eupatoria

1

D.s. Al. Trifolion medii

Trifolium medium

Dactylis glomerata

Galium mollugo

Vicia sepium

Hypericum maculatum

Campanula glomerata

\begin{tabular}{|c|c|c|c|c|c|c|c|c|c|c|c|c|c|c|c|c|c|c|}
\hline 3 & 1 & 4 & 1 & 2 & 5 & 4 & 5 & 5 & 2 & 4 & 3 & . & . & 2 & 2 & 3 & $\mathrm{~V}$ & 14 \\
\hline 2 & 3 & . & 2 & 3 & 2 & 2 & 2 & 1 & 3 & 3 & 2 & 2 & 1 & 2 & 2 & 2 & V & 1 \\
\hline 1 & 2 & 1 & 2 & 1 & 3 & 1 & . & . & 2 & . & . & 1 & 1 & . & 1 & 1 & IV & 1 \\
\hline 1 & . & 1 & . & 1 & . & . & . & . & $\mathrm{r}$ & 2 & 1 & . & . & . & $\mathrm{r}$ & 1 & III & 1 \\
\hline . & . & . & . & . & . & . & . & $\mathrm{r}$ & . & 1 & . & . & $\mathrm{r}$ & . & 1 & 1 & II & . \\
\hline 1 & . & . & . & . & . & . & . & . & . & . & . & . & . & . & 1 & $\mathrm{r}$ & I & . \\
\hline
\end{tabular}




\begin{tabular}{|c|c|c|c|c|c|c|c|c|c|c|c|c|c|c|c|c|c|c|c|c|}
\hline Successive No. of relevé & 1 & 2 & 3 & 4 & 5 & 6 & 7 & 8 & 9 & 10 & 11 & 12 & 13 & 14 & 15 & 16 & 17 & & 18 & 19 \\
\hline No. of relevé in the field & 29 & 17 & 18 & 12 & 37 & 34 & 21 & 20 & 19 & 27 & 23 & 28 & 38 & 11 & 26 & 25 & 24 & & 64 & 36 \\
\hline Shrub layer cover [\%] & 7 & . & 10 & . & 5 & 1 & 5 & . & 10 & 4 & . & 5 & 10 & 5 & 10 & 5 & 10 & $\stackrel{1}{1}$ & 15 & 2 \\
\hline Herb layer cover [\%] & 90 & 95 & 95 & 80 & 70 & 95 & 85 & 100 & 100 & 95 & 95 & 85 & 70 & 65 & 85 & 85 & 95 & $\frac{1}{2}$ & 80 & 85 \\
\hline Moss layer cover[\%] & . & . & . & 5 & . & . & . & . & . & . & . & . & 15 & & & . & 3 & 五 & 10 & 10 \\
\hline Altitude a.s.l. [m] & 178 & 157 & 161 & 170 & 224 & 220 & 200 & 193 & 194 & 184 & 187 & . & 228 & 158 & 186 & 185 & 180 & $\tilde{n}$ & 163 & 172 \\
\hline Inclination $\left[^{\circ}\right]$ & . & $\cdot$ & $\cdot$ & $\cdot$ & $\cdot$ & $\cdot$ & $\cdot$ & . & . & 5 & . & $\cdot$ & $\cdot$ & $\cdot$ & & 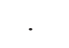 & $\cdot$ & ن & $\cdot$ & $\cdot$ \\
\hline Area of relevé [m2] & 100 & 100 & 50 & 50 & 50 & 20 & 40 & 10 & 60 & 35 & 20 & 25 & 30 & 50 & 25 & 50 & 100 & & 50 & 20 \\
\hline Number of species & 45 & 25 & 30 & 31 & 34 & 32 & 25 & 21 & 29 & 29 & 28 & 33 & 29 & 28 & 30 & 38 & 41 & & 30 & 31 \\
\hline
\end{tabular}

\section{D.s. Cl. Trifolio-Geranietea}

Knautia arvensis

Veronica chamaedrys

Fragaria vesca

Melampyrum nemorosum

Solidago virgaurea

Hieracium umbellatum

Clinopodium vulgare

Hypericum perforatum

Ranunculus polyanthemos

Poa angustifolia

Trifolium alpestre

\begin{tabular}{|c|c|c|c|c|c|c|c|c|c|c|c|c|c|c|c|c|c|c|}
\hline 1 & 1 & . & 1 & 1 & 2 & 2 & $\mathrm{r}$ & . & 1 & $\mathrm{r}$ & $\mathrm{r}$ & 2 & & 1 & 1 & 1 & $\mathrm{~V}$ & 3 \\
\hline 2 & 1 & 1 & 1 & 2 & 1 & 1 & 1 & . & 1 & 2 & . & 1 & 1 & & 2 & 2 & $\mathrm{~V}$ & 1 \\
\hline 2 & 1 & . & 1 & 2 & 1 & . & . & . & . & . & 1 & & 1 & 1 & 1 & 2 & III & 4 \\
\hline . & . & 2 & 1 & 2 & . & 1 & 2 & 1 & 3 & 3 & 2 & 3 & 4 & 1 & 1 & 2 & $\mathrm{~V}$ & 3 \\
\hline 1 & . & 1 & 1 & 1 & 1 & . & . & . & $\mathrm{r}$ & . & & 1 & 1 & 1 & 1 & 1 & IV & . \\
\hline . & . & 1 & $\mathrm{r}$ & . & . & 2 & $\mathrm{r}$ & . & . & . & . & . & $\mathrm{r}$ & 2 & 1 & 2 & III & . \\
\hline . & . & . & . & . & . & . & . & . & . & . & . & 2 & $\cdot$ & $\cdot$ & $\cdot$ & . & I & . \\
\hline 1 & . & . & . & $\mathrm{r}$ & . & . & . & . & . & . & . & $\mathrm{r}$ & . & . & . & r. & I & . \\
\hline . & . & . & . & . & . & . & . & . & . & . & . & $\mathrm{r}$ & . & . & 2 & . & I & 1 \\
\hline 3 & 2 & . & . & . & 2 & . & 2 & . & . & . & . & . & . & $\cdot$ & . & . & II & . \\
\hline . & . & . & . & . & . & . & . & . & . & . & $\mathrm{r}$ & . & . & 2 & . & . & I & . \\
\hline
\end{tabular}

D.s. Cl. Molinio-Arrhenateretea

Phleum pratense

Equisetum pratense

Rumex acetosa

Taraxacum officinale

Schenodorus pratensis

Poa pratensis

Trifolium repens

Vicia cracca

Centaurea jacea

Leucanthemum vulgare

Plantago lanceolata

Alchemilla sp.

Ranunculus acris

Stellaria graminea

Lathyrus pratensis

Medicago lupulina

Trifolium pratense

Leontodon automnalis

D.s. Cl. Vaccinio-Piceetea

Luzula pilosa

Trientalis europaea

Maianthemum bifolium

Vaccinium vitis-idaea

Hylocomium splendens d

D.s. Cl. Carpino-Fagetea

Corylus avellana b

Hepatica nobilis

Stellaria holostea

Asarum europaeum

Viola riviniana

Acer platanoides b

Fraxinus excelsior b

Primula veris

Carex pilosa

Phyteuma spicatum

D.s. Cl. Nardetea strictae

Potentilla erecta

Veronica officinalis 
Carex pallescens

D.s. Cl. Festuco-Brometea

Centaurea scabiosa

Medicago falcata

Trifolium montanum

D.s. Cl. Epilobietea

Aegopodium podagraria

Anthriscus sylvestris

Geum urbanum

Heracleum sphondylium s.l.

Urtica dioica

Galeopsis tetrahit

D.s. Cl. Artemisietea

Artemisia vulgaris

Elytrigia repens

Tanacetum vulgare

Silene latifolia

Cichorium intybus

Tussilago farfara

Melilotus albus

D.s. O. Molinietalia caerulea

Deschampsia caespitosa

Angelica sylvestris

Other species

Agrostis capillaris

Pimpinella saxifraga

Achillea millefolium

Cirsium arvense

Quercus robur b

Quercus robur c

Medicago sativa

Rubus idaeus

Carex hirta

Equisetum sylvaticum

Ranunculus repens

Populus tremula b

Ajuga reptans

Malus domestica $\mathrm{b}$

Sorbus aucuparia b

Silene vulgaris

Alnus glutinosa b

Calamagrostis epigeios

Lupinus polyphyllus

Carex contigua

Bromopsis inermis

Sonchus arvensis

Rumex sanguineus

Filipendula ulmaria

Geranium palustre

Salix caprea b

Salix cinerea $\mathrm{b}$

Dianthus barbatus

Pteridium aquilinum

Prunella vulgaris

Platanthera bifolia

Calamagrostis arundinacea

$\begin{array}{llll}2 & 2 & . & 1 \\ . & 2 & 2 & . \\ 1 & . & 1 & .\end{array}$


Lathyrus sylvestris (r), Lysimachia vulgaris (1); rel. 6: Festuca ovina (r); rel. 7: Epipactis helleborine (1); rel. 9: Campanula patula (r), Galeopsis bifida (1), Impatiens noli-tangere (1), Stachys palustris (r), Trifolium hybridum (r); rel. 11: Athyrium filix-femina (r); rel. 12: Pulmonaria obscura (r); rel. 13: Anthoxanthum odoratum (2), Dicranum polysetum d (1), Orthilia secunda (1), Pleurozium schreberi d (2), Polemonium caeruleum (r), Viola canina (r); rel. 14: Eupatorium cannabinum (r), Moehringia trinervia (1), Prunus padus b (1), Ribes sp. b (1), Vaccinium myrtillus (1); rel. 16: Stachys officinalis (2), Campanula persicifolia (1), Selinum carvifolia (1), Succisa pratensis (r); rel. 17: Juncus conglomeratus (r), Nardus stricta (1), Ptilium crista-castrensis d (1); rel. 18: Chelidonium majus (r), Oxalis acetosella (2); rel. 19: Carex digitata (1), Melica nutans (r), Stellaria media (r).

List of localities:

1 - 54도. $582^{\prime}$ N $26^{\circ} 26.766^{\prime} \mathrm{E}$; Minsk Region, Miadziel district, vicinities of Kanstantinava village; 15.08 .2012 ; 2 - 54 ${ }^{\circ} 54.432^{\prime}$ N 26 $6^{\circ} 43.811^{\prime}$ E; Minsk Region, Miadziel district, vicinities of Narach town; 13.08.2012; 3-5458.108' N 26 $6^{\circ} 48.275^{\prime} \mathrm{E}$; Minsk Region, Miadziel district, vicinities of Chuchelitsy

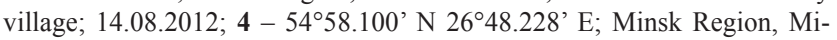
adziel district, vicinities of Chuchelitsy village; $14.08 .2012 ; \mathbf{5}-54^{\circ} 57.955^{\text {, }}$ N 26⒋305' E; Minsk Region, Miadziel district, vicinities of Chuchelitsy

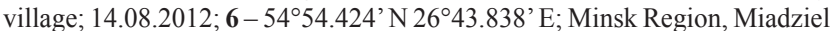
district, vicinities of Narach town; 13.08 .2012 ; 7 - 54 $54.549^{\prime} \mathrm{N} 26^{\circ} 26.750^{\prime}$ E; Minsk Region, Miadziel district, vicinities of Kanstantinava village; 15.08.2012; 8 - 54 ${ }^{\circ} 56.942^{\prime} N$ 26²9.292' E; Minsk Region, Miadziel district, vicinities of Chuchelitsy village; $14.08 .2012 ; 9-54^{\circ} 57.188^{\prime} \mathrm{N} 26^{\circ} 48.682^{\prime} \mathrm{E}$; Minsk Region, Miadziel district, vicinities of Chuchelitsy village; 14.08.2012; 10 - 54 $57.200^{\prime}$ N 26 $48.733^{\prime}$ E; Minsk Region, Miadziel district, vicinities

forests. Average number of species per relevé - 31.2. Diagnostic species: Melampyrum nemorosum (dom., opt.), Trifolium medium (dom., opt.).

Trifolio medii-Agrimonietum T. Müller 1962 association (Table 4, rel. 18-19): Relatively dense (total cover $80-85 \%$ ) communities with significant participation of Agrimonia eupatoria (15-20\%) and other species of the Trifolion medii alliance (Trifolium medium, Knautia arvensis, Galium mollugo, etc.). Moss layer (cover $10 \%$ ) was formed mainly by Hylocomium splendens. These communities were described on mesophilous margins of the nemoral spruce forests. Average number of species per relevé comprised 30.5. Diagnostic species: Agrimonia eupatoria (opt., dom.).

\section{Discussion}

The positions of thermophilous xero-mesophytic forb-fringe communities with Geranium sanguineum in the Baltic region are widely discussed (Westhoff et al. 1983; Dierssen \& Dierssen 1996; Rūsiña 2007; Dengler \& Boch 2008). We described these communities close to the southern limit of hemiboreal zone. According to chorological data, some thermophilous fringe species have specific distribution patterns in the territory of Belarus (Kozlovskaya \& Parfenov 1972; Kachanovskiy et al. 2015): such species as Clematis recta, Hypericum montanum, Peucedanum cervaria, Potentilla alba, Pyrethrum corymbosum, Stachys recta, considered as characteristic species of the Geranion sanguinei alliance and, partly, of the Geranio-Trifolietum alpestris T. Müller 1962 association, are limited in their distribution mainly to southern regions of the country. So, we could not expect these species on forest edges of Chuchelitsy village; $14.08 .2012 ; 11$ - 54 $4^{\circ} 56.343^{\prime}$ N 26²4.420' E; Minsk Region, Miadziel district, vicinities of Grumbinenty village; 15.08.2012; 12 - 54 ${ }^{\circ} 57.914^{\prime}$ N $26^{\circ} 48.297^{\prime}$ E; Minsk Region, Miadziel district, vicinities of Chuchelitsy village; $14.08 .2012 ; 13-54^{\circ} 57.866^{\prime} \mathrm{N} 26^{\circ} 48.315^{\prime} \mathrm{E}$; Minsk Region, Miadziel district, vicinities of Chuchelitsy village; 14.08.2012; 14 - 54 ${ }^{\circ} 58.155^{\prime}$ N 26 $6^{\circ} 48.349^{\prime}$ E; Minsk Region, Miadziel district, vicinities of Chuchelitsy village; $14.08 .2012 ; 15-54^{\circ} 58.167^{\prime}$ N 26 $6^{\circ} 48.350^{\prime}$ E; Minsk Region, Miadziel district, vicinities of Chuchelitsy village; 14.08.2012; 16 - 54 $4^{\circ} 58.125^{\prime} \mathrm{N} 26^{\circ} 48.340^{\prime} \mathrm{E}$; Minsk Region, Miadziel district, vicinities of Chuchelitsy village; $14.08 .2012 ; 17-54^{\circ} 58.188^{\prime}$ N 26 $6^{\circ} 48.438^{\prime}$ E; Minsk Region, Miadziel district, vicinities of Chuchelitsy village; 14.08.2012; $18-54^{\circ} 54.437^{\prime} \mathrm{N} 26^{\circ} 43.522^{\prime} \mathrm{E}$; Minsk Region, Miadziel district, vicinities of Narach town; $17.08 .2012 ; 19-54^{\circ} 57.628^{\prime} \mathrm{N} 26^{\circ} 21.869^{\prime} \mathrm{E}$; Minsk Region, Miadziel district, vicinities of Glublia lake; 15.08.2012

of hemiboreal zone, and, consequently, thermophilous fringes communities with Geranium sanguineum on the northern part of Belarus could not be classified as Geranio-Trifolietum alpestris association (confront with preliminary communication by Iakushenko \& Tsvirko 2013).

In contrast, localities of some thermophilous species noticed in the phytosociological relevés on this survey (i.e. Dracocephalum ruyschiana, Laserpitium latifolium Lathyrus pisiformis) were concentrated mainly (but not exclusively) on highlands in the central part of the country (Kozlovskaya \& Parfenov 1972; Kachanovskiy et al. 2015).

For Dracocephalum ruyschiana, in accordance with the chorological data, the Baltic fragment of the European part of the species range could be recognized: it includes South Scandinavia, North-Eastern Poland, Baltic States, and Central Belarus (Hultén \& Fries 1986; Zając \& Zając 2001; Yakovleva 2015). In this area, current localities of the discussed heliophilous species were mostly related to the thermophilous sites occupied by the communities of the Trifolio-Geranietea class or to the sparse subcontinental species-rich Scots pine forests (Bambe 2003; Patalauskaite 2007; Adamowski \& Wołkowycki 2014). We wondered, whether communities with D. ruyschiana from the northern Belarus belong to the association Origano-Dracocephaletum ruyschianae Kielland-Lund 1965 , also supposed to be recorded in northern Poland (Brzeg 2005), and found that this name was rejected as invalid. These communities were merged with the association Galio borealis-Geranietum sanguinei Tüxen 1967 (Dengler \& Boch 2008).

As for Lathyrus pisiformis L., only few isolated populations are known currently in Belarus, also in the 
central and in the northern parts (Savchuk \& Semerenko 2015). The species prefers edges and glades in the sparse species-rich Scots-pine forests on the steep slopes of moraine hills. In the same conditions, L. pisiformis was recorded in north-eastern Poland (Pawlikowski 2005; Herbich \& Łazarski 2014), Lithuania (Čiuplys 2007), and Latvia (Bambe 2003).

Another fringe species, Laserpitium latifolium, was co-dominant in the described phytocoenoses on Minskaya highland. Despite the proposal to put northEuropean communities with L. latifolium into Trifolio medii-Laserpitietum latifolii van Gils \& Gilissen 1976 association (Passarge 1979; Dengler \& Boch 2008), the author supports the opinion of Diekmann (1990) and merged them with the Galio borealis-Geranietum sanguinei Tüxen 1967 association. Moreover, it should be admitted, the occurrence of the last association in Belarus is quite possible, taking into consideration literature data of the species distribution and its habitats (Kozlovskaya \& Parfenov 1972; Chernik \& Dzhus 2011; Skuratovich 2015). Thus, we suggest the described communities with significant role of Geranium sanguineum in the hemiboreal part of Belarus belong to the association Galio borealis-Geranietum sanguinei Tüxen 1967, on the southern limit of its range.

The edge communities on nutrient-poor acidophilous margins of Scots-pine forests dominated by Melampyrum pratense belong to the Melampyrion pratensis Passarge 1979 alliance. The position of communities described in this article is still unclear on the association level. In Central Europe, the Lathyro montaniMelampyretum pratensis Passarge 1967 association was described (Passarge 1994), later the range of this association was extended to Eastern Poland: few localities are known between the Vistula and Bug rivers (Brzeg 2005). In the study area, Melampyrum pratense was a common species on edges of coniferous forests, but it was hard to find another vascular plant species characteristic for these fringe communities (perhaps, because of insufficient amount of relevés in our dataset). Typical species for acidophilous forest edges, Holcus mollis, was spread sporadically in southern, western and central regions of the country, and occurred much rarer - in northern and eastern regions (Tretyakov et al. 2013). In Belarus, Lathyrus montanus reached the eastern limit of the range, its localities were concentrated mainly on moraine highlands in the central part of the country, and this species was absent in Narachanskaya plain (Kozlovskaya \& Parfenov 1972; Semerenko $\&$ Savchuk 2015), where the analyzed relevés were sampled. On the other hand, we could assume some of the numerous species from the Pilosella Vaill. genus occurring in Belarus (Tikhomirov 2000) might be assigned a high fidelity threshold in acidophilous fringe communities associated with pine forests margins. Thus, the described community with Melampyrum pratense might be recognized further as specific geographical variant of the Lathyro montani-Melampyretum pratensis association.

Two stands with Vicia cassubica domination, according to the results of cluster analysis, were closer to Melampyrion pratense communities than to Trifolion medii communities: these 2 plots (group 3 ) were located within the group of the communities with Melampyrum pratense (group 2), but not within the group of relevés belonging to Trifolion medii (groups 4-6) (Fig. 2). Besides, this species is considered to be characteristic for the mesophilous Agrimonio-Vicietum cassubicae Passarge 1967 association, it appeared regularly in other more or less acidophilous, xero-mesotermic forest-fringe communities (Brzeg 2005). So, we suggest these stands, which were only patches limited by the coenopopulation of one dominant species, belong to the Melampyrion pratensis alliance due to floristic composition and ecological conditions. At the current stage of research, it is hard to determine syntaxonomical positions of these communities more precisely.

The mesophilous fringe communities of the Trifolion medii alliance were mentioned previously for the north-western part of Belarus, where the Trifolietum medii Stepanovič (1987) 1991 association was described (Scepanovich \& Scepanovich 1991; Scepanovich 2000). This name should be rejected according to article 31 of the International Code of Phytosociological Nomenclature (Weber et al. 2000). Later, the same author used the form Trifolietum medii Müller $1961 \mathrm{em}$. Stepanovič (1987) 1991 (Scepanovich 2006), which should also be rejected as a homonym.

Communities of the Trifolio-Melampyretum nemorosi association are very common in north-western part of Belarus, and seem to be widespread in whole territory of the country. This association was mentioned for Belarus as Stachyo-Melampyretum nemorosi Passarge 1967 (Scepanovich 2006). We did not find significant differences within the analyzed dataset between stands dominated by Melampyrum nemorosum or Trifolium medium, so we did not support the split of such communities into 2 different associations. Taking into consideration the restricted number of relevés involved in this survey, we confined deliberately the attempts to recognize subassociations, because phytosociological materials are scarce and not sufficient for such analyses. Differentiation of this association into subassociations should be the aim of further investigation.

As the field survey was mainly concentrated on forest margins, only two phytosociological relevés of semi-synanthropic communities with significant role of Agrimonia eupatoria were conducted. These coenoses were classified as Trifolio medii-Agrimonietum $\mathrm{T}$. Müller 1962 association. For better delimitation from 
the previous association and precise determination of distribution patterns, wide survey of mesophilous forbfringe vegetation on larger area is needed.

\section{Conclusion}

Three associations and one community from the Trifolio-Geranietea sanguinei T. Müller 1962 class were distinguished as a result of a short phytosociological survey on forest margins and glades in Belarus. One association, Galio borealis-Geranietum sanguinei Tüxen 1967, was mentioned for the first time for this area. The conducted study enriched the syntaxonomical scheme of the vegetation of Belarus, but the number of associations known from this territory is still inadequate to species pool, forest vegetation diversity and potential assemblages. We strongly believe that forest edge communities in Belarus are much more diverse, and the presented article is only a small contribution to their description and evaluation.

Aknowledgements. The author is grateful to Ruslan Tsvirko (Insitute of Experimental Botany, National Academy of Sciences of Belarus, Minsk) for his hospitality and support during field research. We warmly thank the anonymous reviewers for the careful reading of the manuscript and for their critical comments and suggestions.

\section{References}

Adamowski W. \& Wolkowycki D. 2014. Dracocephalum ruyschiana L. Pszczelnik waskolistny. In: R. KaźmiercZakowa, K. ZARZYcKi \& Z. Mirek (eds.). Polska czerwona księga roślin. Paprotniki i rośliny kwiatowe. Wydanie III uaktualnione i rozszerzone, pp. 425-427. Instytut Ochrony przyrody PAN, Kraków.

Balevičiene J., Kiziene B., Lazdauskaite Ž., Patalauskaite D., Rašomavičıus V., SinkevičIene Z., Tučiene A. \& Venckus Z. 1998. Lietuvos Augalija I. Pievos. 269 pp. Šviesa, Kaunas-Vilnius

BAmBe B. 2003. Pine forest plant communities in the Daugava Loki Nature Park. Acta Universitatis Latviensis, Earth and Environment Sciences 654: 64-98.

Bohn U., Golub G. \& Hettwer C. 2000. Karte der natürlichen Vegetation Europas - Masstab 1:2 500000 - Legende. 153 pp. Bundesamt für Naturschutz, Bonn.

BRzEG A. 2005. Zespoły kserotermofilnych ziołorośli okrajkowych z klasy Trifolio-Geranietea sanguinei Th. Müller 1962 w Polsce. 236 pp. Bogucki Wyd. Nauk., Poznań.

Brzeg A. \& WiKa S. 2014. Termofilne ziołorośla okrajkowe z klasy Trifolio-Geranietea sanguinei Th. Müller 1962 na obszarze środkowej części Wyżyny Krakowsko-Częstochowskiej (wyd. II poprawione). 179 pp. Zespół Parków Krajobrazowych Województwa Śląskiego, Katowice.

Chernik V. V. \& Dzhus M. A. 2011. Kompleksy redkikh i okhranyaemykh vidov rasteniy tsentralnogo regiona Belorusskoy gryady. Vestn. BGU, Ser. 2(1): 54-59.

ChytrÝ M. (ed.). 2007. Vegetace České republiky. 1. Travinná a keříčková vegetace. 526 pp. Academia, Praha.

Chytrý M., TichÝ M., Holt J. \& Botta-Dukát Z. 2002. Determination of diagnostic species with statistical fidelity measures. Journal of Vegetation Science 13: 79-90.

ČARNI A. 2005. Trifolio-Geranietea vegetation in south and southeast Europe. Acta Botanica Gallica 152(4): 483-496.

ČIUPLYs R. 2007. Žirnialapis pelëžirnis Lathyrus pisiformis L. In: V. RašomaviČIUs (ed.). Lietuvos raudonoji knyga - Red Data Book of Lithuania. 462 pp. Lutute, Kaunas.

Dengler J. \& Boch S. 2008. Forest-edge communities (Trifolio-Geranietea sanguinei) on the island of Saaremaa (Estonia): Phytosociology and biodiversity patterns. In: J. Dengler, C. Dolnik \& M. Trepel (eds.). Flora, Vegetation, and Nature Conservation from SchleswigHolstein to South America - Festschrift for Klaus Dierßen on Occasion of his 60th Birthday. Mitt. Arbeitsgem. Geobot. Schleswig-Holstein Hamb. 65: 257-285.

Diekmann M. 1990. Saumgesellschaften aufÖland. Tuexenia 10: 349-367.

DierschKe H. 1974a. Saumgesellschaften im Vegetations- und Standortsgefälle an Waldrändern. Scripta Geobotanica 6: 1-225. Verlag Erich Goltze KG, Göttingen.

Dierschke H. 1974b. Zur Syntaxonomie der Klasse TrifolioGeranietea. Mitt. Florist.-Soziol. Arbeitsgem. N. F. 17: 27-38.

Dierssen K. \& Dierssen B. 1996. Vegetation Nordeuropas. 838 pp. Ulmer, Stuttgart.

Euro+Med. 2006-. Euro+Med PlantBase - the information resource for Euro-Mediterranean plant diversity. Published on the Internet http://ww2.bgbm.org/EuroPlusMed/ [accessed 12.09.2016].

Evans D. 2005. Some Uses of the Map of the Natural Vegetation of Europe for Natura 2000. In: U. BoHn, C. Hettwer \& G. Gollub (eds.). Anwendung und Auswertung der Karte der natürlichen Vegetation Europas/ Application and Analysis of the Map of the Natural Vegetation of Europe, pp. 195-204. Bundesamt für Naturschutz, Bonn.

Hennekens S. M. \& Schaminée J. H. J. 2001. Turboveg, a comprehensive data base management system for vegetation data. J Veg Sci 12: 589-591.

Herbich J. \& Lazarski J. 2014. Lathyrus pisiformis L. Groszek wielkoprzylistkowy. In. R. KAźmIERCZAKOwA, K. ZARZYCKI \& Z. Mirek (eds.). Polska czerwona księga roślin. Paprotniki i rośliny kwiatowe. Wydanie III uaktualnione i rozszerzone, pp. 291-293. Instytut Ochrony Przyrody PAN, Kraków. 
Hill M. O., Bell N., Bruggeman-Nannenga M. A., Brugués M., Cano M. J., Enroth J., Flatberg K. I., Frahm J.P., Gallego M. T., Garilleti R., Guerra J., Hedenäs L., Holyoak D. T, Hyvőnen J., Ignatov M. S., Lara F., Mazimpaka V., Muñoz J. \& Söderström L. 2006. An annotated checklist of the mosses of Europe and Macaronesia. J Bryol 28: 198-267.

Hultén E. \& Fries M. 1986. Atlas of North European vascular plants. North of the Tropic of Cancer. I-III. xvi+1172 pp. Koeltz Scientific Books, Königstein.

IAKUSHENKo D. \& Tsvirko R. 2013. Travyanistye opushechnye soobschestva natsionalnogo parka "Narochanskiy". In.: Aktualnye problemy izucheniya i sokhraneniya fito- i mikobioty. Sbornik statey II mezhdunarodnoy nauch.-prakt. konf., pp. 85-87. Izdatelskiy tsentr BGU, Minsk.

Kozlovskaya N.V. \& PARfenov V.I. 1972. Khorologiya flory Belorussii. 312 pp. Nauka i tekhnika, Minsk.

Kachanovskiy I. M., Nikiforov M. Ye., Parfenov V. I., Borodin O. I., Pugachevskiy A. V., Baychorov V. M., Gapiyenko O. S., Giryachev A. S., Yevdaseva T. P. (eds.). 2015. Krasnaya kniga Respubliki Belarus, rasteniya: redkiye i nakhodyashchiyesya pod ugrozoy ischeznoveniya vidy dikorastushchikh rasteniy, 4. ed., 448 pp. Belaruskaya Entsyklapedyya imya P. Broy̆ki, Minsk, (in Russian).

Lukash O. \& IAKushenko D. 2008. Asotsiatsiia GeranioTrifolietum alpestris Th. Müller 1962 na Skhidnomu Polissi. Ukr Bot J 65(3): 336-350.

McCune B. \& Mefford M. J. 1999. PC-ORD. Multivariate Analysis of Ecological Data. version 4.

Mucina L. 1997. Conspectus of classes of European vegetation. Folia Geob Phytotx 32: 117-172.

Mucina L., Bültmann H., Dierssen K., Theurillat J.-P., Raus T., Čarni A., Šumberová K., Willner W., Dengler J., Gavilán García R., Chytrý M., Hájek M., Di Pietro R., Iakushenko D., Pallas J., Daniëls F. J. A., Bergmeier E., Santos Guerra A., Ermakov N., Valachovič M., Schaminée J. H. J., Lysenko T., Didukh Y. P., Pignatti S., Rodwell J. S., Capelo J., Weber H. E., Solomeshch A., Dimopoulos P., Aguiar C., Hennekens S. M. \& TichÝ L. 2016. Vegetation of Europe: hierarchical floristic classification system of vascular plant, bryophyte, lichen, and algal communities. App Veg Sci 19 (Supplement 1): 3-264.

Mucina L. \& Kolbek J. 1993. Trifolio-Geranietea sanguinei. In: L. Mucina, G. Grabherr \& T. Ellmauer (eds.). Die Pflanzengesellschaften Österreichs. Teil I, pp. 252-270. Gustav Fischer, Jena.

MüLler Th. 1962. Die Saumgesellschaften der Klasse Trifolio-Geranietea sanguinei. Mitt. Flor-soz. Arbeitsgem., N. F. 9: 95-140.

MyASNiKovich M. U. (ed.). 2002. Natsyyanalny atlas Belarusi. 292 pp. Belkartografiya, Minsk (in Russian).

Passarge H. 1979. Über vikariierende Trifolio-GeranieteaGesellschaften in Mitteleuropa. Feddes Repertorium 90(1-2): 51-83.

Passarge H. 1994. Azidophile Waldsaum-Gesellschaften (Melampyro-Holcetea mollis) in europäischen Raum. Tuexenia 14: 83-111.
Patalauskaitė D. 2007. Miškinė žiomenė Dracocephalum ryuschiana L. In: V. RAšomavičIUS (ed.). Lietuvos raudonoji knyga - Red Data Book of Lithuania, pp. 510. Lututè, Kaunas.

PAWLikowski P. 2005. Nowe stanowisko Lathyrus pisiformis (Fabaceae) na Pojezierzu Litewskim. Fragm. Flor. Geobot. Polonica 12(1): 168-171.

Rūsıña S. 2007. Latvijas mezofīto un kserofīto zālāju daudzveidība un kontaktsabiedrības. Latvijas Veǵetācija 12: 1-366.

Savchuk S. S. \& Semerenko L. V. 2015. Lathyrus pisiformis L. In: I. M. Kachanovskiy, M. Ye. Nikiforov, V. I. Parfenov, O. I. Borodin, A. V. Pugachevskiy, V. M. Baychorov, O. S. Gapiyenko, A. S. Giryachev \& T. P. Yevdaseva (eds.). 2015. Krasnaya kniga Respubliki Belarus, rasteniya: redkiye i nakhodyashchiyesya pod ugrozoy ischeznoveniya vidy dikorastushchikh rasteniy, 4. ed., 114-115 pp. Belaruskaya Entsyklapedyya imya P. Broy̆ki, Minsk, (in Russian).

ScePANOvich I. M. 2000. Ekolaga-flarystychny dyyagnaz sintaksonau pryrodnay travyanistay raslinnastsi Belarusi. 140 pp. Kamtat, Minsk.

Scepanovich I. M. 2006. Fitatsenaraznastaynasts raslinnastsi Belarusi. Botanika (issledovaniya) 34: 264-281.

Scepanovich I. M. \& Scepanovich A. F. 1991. Travyanistyya supolnitstvy na myazhy lug-les va umovakh paunochnaha zakhadu Belarusi. Vesci Akademii Navuk Belaruskay SSR 2: 3-14.

Semerenko L. V. \& SAVChuK S. S. 2015. Lathyrus linifolius (Reinchard) Bässler. In: I. M. KachanovskiY, M. Ye. Nikiforov, V. I. Parfenov, O. I. Borodin, A. V. Pugachevskiy, V. M. Baychorov, O. S. Gapiyenko, A. S. Giryachev \& T. P. Yevdaseva (eds.). 2015. Krasnaya kniga Respubliki Belarus, rasteniya: redkiye i nakhodyashchiyesya pod ugrozoy ischeznoveniya vidy dikorastushchikh rasteniy, 4. ed., 233-234 pp. Belaruskaya Entsyklapedyya imya P. Broy̆ki, Minsk, (in Russian).

SkURatovich A. N. 2015. Laserpitium latifolium L. In: I. M. Kachanovskiy, M. Ye. Nikiforov, V. I. Parfenov, O. I. Borodin, A. V. Pugachevskiy, V. M. Baychorov, O. S. Gapiyenko, A. S. Giryachev \& T. P. Yevdaseva (eds.). 2015. Krasnaya kniga Respubliki Belarus, rasteniya: redkiye i nakhodyashchiyesya pod ugrozoy ischeznoveniya vidy dikorastushchikh rasteniy, 4. ed., 178-179 pp. Belaruskaya Entsyklapedyya imya P. Broy̆ki, Minsk, (in Russian).

Solomakha V. A. 2008. Syntaksonomiia roslynnosti Ukrajiny. Tretie nablyzhennia. 296 pp. Fitosociocentr, Kyiv.

TICHÝ L. 2002. JUICE, software for vegetation classification. J Veg Sci 13: 451-453.

Tiknomirov V.N. 2000. Rod Pilosella (Asteraceae) vo flore Belarusi. Bot. zhurn. 85(11): 104-114.

Tretyakov D. I., Dubovik D. V., Skuratovich A. N., Parfenov V. I., Yakovleva I. M., Lebedko V. N., Bednarskaya I. A., Lazarevich S. V., Zhytenyev L. A. 2013. Flora Belarusi. Sosudistye rasteniya. 2. Liliopsida (Acoraceae, Alismataceae, Araceae, Butomaceae, Commelinaceae, Hydrocharitaceae, Juncaginaceae, Lemnaceae, Najadaceae, Poaceae, 
Potamogetonaceae, Scheuchzeriaceae, Sparganiaceae, Typhaceae, Zannichelliaceae). 447 pp. Belaruskaya navuka, Minsk.

Valachovič M. \& Hegedüšová Vantarová K. 2014. TrifolioGeranietea. In: K. HedegüŠová Vantarová \& I. ŠKoDOvá (eds.). Rastlinné spoločenstvá Slovenska. 5. Travinno-bylinná vegetácia, pp. 149-190. Veda, Bratislava.

Weber H. E., Moravec J. \& Theurillat J.-P. 2000. International code of the phytosociological nomenclature. 3rd edition. J Veg Sci 11: 739-768.

Westhoff V. \& van der MaArel E. 1973. The Braun-Blanquet approach. 2-nd ed. In: R. WhITTAKER (ed.). Classification of plant Communities, pp. 287-399. Junk, The Hague.

Westhoff V., Schaminée J. H. J. \& Sýkora K. V. 1983. Aufzeichungen zur Vegetation der schwedischen Inseln Öland, Gotland und Stora Karlsö. Tuexenia 3: 179-198.
YAKovleVA I. M. 2015. Dracocephalum ruyschiania L. In: I. M. Kachanovskiy, M. Ye. Nikiforov, V. I. Parfenov, O. I. Borodin, A. V. Pugachevskiy, V. M. Baychorov, O. S. Gapiyenko, A. S. Giryachev \& T. P. Yevdaseva (eds.). 2015. Krasnaya kniga Respubliki Belarus, rasteniya: redkiye i nakhodyashchiyesya pod ugrozoy ischeznoveniya vidy dikorastushchikh rasteniy, 4. ed., 125-126 pp. Belaruskaya Entsyklapedyya imya P. Broy̆ki, Minsk, (in Russian).

Yurkevich I. D., Golod D. S. \& Aderikho V. S. 1979. Rastitel'nost' Belorussii, ee kartografirovanie, okhrana i ispolzovanie. 248 pp. Nauka i technika, Minsk.

Zając A. \& ZająC M. (eds.). 2001. Distribution Atlas of Vascular Plants in Poland. xii+714 pp. Edited by Laboratory of Computer Chorology, Institute of Botany, Jagiellonian University, Cracow. 